\title{
Overexpression of a novel candidate oncogene KIF14 correlates with tumor progression and poor prognosis in prostate cancer
}

\author{
Yixiang Zhang ${ }^{1, *}$, Yeqing Yuan ${ }^{1, *}$, Pei Liang ${ }^{2, *}$, Zhaoxia Zhang ${ }^{3}$, Xiaojing Guo ${ }^{4}$, Ligang \\ $X{ }^{5}{ }^{5}$, Yingying Zhao ${ }^{6}$, Xing-Sheng Shu ${ }^{7}$, Shengkun Sun ${ }^{8}$, Ying Ying $^{6}$ and Yingduan Cheng ${ }^{1}$ \\ 1Department of Urology, The Second Affiliated Hospital of Jinan University, Shenzhen People's Hospital, Shenzhen, \\ Guangdong, People's Republic of China \\ ${ }^{2}$ Department of Urology, David Geffen School of Medicine, University of California Los Angeles, Los Angeles, California, USA \\ ${ }^{3}$ Department of Pediatrics, The Second Affiliated Hospital of Jinan University, Shenzhen People's Hospital, Shenzhen, \\ Guangdong, People's Republic of China \\ ${ }^{4}$ Department of Pathology, The Second Affiliated Hospital of Jinan University, Shenzhen People's Hospital, Shenzhen, \\ Guangdong, People's Republic of China \\ ${ }^{5}$ Department of Gastrointestinal Surgery, The Second Affiliated Hospital of Jinan University, Shenzhen People's Hospital, \\ Shenzhen, Guangdong, People's Republic of China \\ ${ }^{6}$ Department of Physiology, School of Basic Medical Sciences, Shenzhen University Health Sciences Center, Shenzhen, \\ Guangdong, People's Republic of China \\ ${ }^{7}$ Institute of Molecular Medicine, Health Science Center, Shenzhen University, Shenzhen, Guangdong, People's Republic of China \\ ${ }^{8}$ Department of Urology, Chinese PLA General Hospital, Beijing, People's Republic of China \\ *These authors contributed equally to this work
}

Correspondence to: Yingduan Cheng, email: chengyingduan@gmail.com

Ying Ying, email: yingying@szu.edu.cn

Keywords: KIF14, prostate cancer, apoptosis, proliferation, G2 arrest

Received: March 09, 2017 Accepted: April 18, $2017 \quad$ Published: May 02, 2017

Copyright: Zhang et al. This is an open-access article distributed under the terms of the Creative Commons Attribution License 3.0 (CC BY 3.0), which permits unrestricted use, distribution, and reproduction in any medium, provided the original author and source are credited.

\section{ABSTRACT}

Prostate cancer (PCa) is the second leading cause of death from cancer in men. The mechanism underlying tumorigenesis and development of PCa is largely unknown. Here, we identified Kinesin family member 14 (KIF14) as a novel candidate oncogene in PCa. We found that KIF14 was overexpressed in multiple PCa cell lines and primary PCa tissues. Knockdown of KIF14 in DU145 and PC3 prostate cancer cells suppressed cell proliferation, induced cell cycle arrest and apoptosis. Transcriptome analysis by RNA-sequencing demonstrated that KIF4 suppression led to transcriptional changes of genes involved in p53 and TGF-beta signaling pathway. In addition, upregulated expression of GADD45A, GADD45B, p21, PIDD and Shisa5, which contribute to growth arrest and apoptosis induction, and downregulated CCNB1 that promotes cell cycle progression were confirmed by quantitative real-time PCR after KIF4 knockdown. We further found that KIF14 protein level was positively correlated with $\mathrm{T}$ stage and Gleason Score. Patients with higher KIF14 expression had shorter overall survival time than those with lower KIF14 expression. Thus, our data indicate that KIF14 could act as a potential oncogene that contributes to tumor progression and poor prognosis in $\mathrm{PCa}$, which may represent a novel and useful prognostic biomarker for PCa.

\section{INTRODUCTION}

Prostate cancer $(\mathrm{PCa})$ is one of the most commonly diagnosed malignant tumor and the second leading cause of cancer death among men in the United States. In 2016, about 180,890 new PCa cases were diagnosed and 26,120
PCa deaths were occurred in USA [1]. About one-third of patients with organ-confined PCa fail surgical treatment and progress to aggressive or metastatic lethal disease in 10 years [2]. Thus, understanding mechanisms underlying the tumorigenesis and development of $\mathrm{PCa}$ is urgently needed for early diagnosis, risk stratification and selection 
of therapy strategies against PCa. However, despite the impressive progress in discovery of genomic alterations in $\mathrm{PCa}$, novel genomic bio-markers and proteins still remain to be identified.

The kinesin family proteins (KIFs) are ATP- and microtubule-associated motor proteins involved in cell division, microtubule polymer dynamics, intracellular transportation and signal transduction [3]. Kinesin family member 14 (KIF14) contains a C-terminal citron kinase binding region and an $\mathrm{N}$-terminal protein regulating cytokinesis binding region $[4,5]$, and was found to bind to chromatin and microtubules during the formation of the bipolar spindle [4, 6]. Elevated KIF14 expression can induce rapid and error-prone mitosis, leading to aneuploidy during tumorigenesis [6]. Although the exact molecular functions of KIF14 are still under investigation, increasing evidences has implicated KIF14 as an oncogene whose overexpression has been found in multiple types of tumors, including hepatocellular carcinoma, lung cancer, breast cancer, glioma, laryngeal carcinoma and ovarian cancer, etc [6-12]. Moreover, KIF14 levels are prognostic for the outcome of hepatocellular carcinoma and ovarian cancer, where KIF14 overexpression enhances tumor growth, while its knockdown decreases tumorigenicity in vitro and in xenografts [13]. However, the expression of KIF14 and its role in prostate carcinoma has not been reported thus far.

In this study, we found that KIF14 was upregulated in human $\mathrm{PCa}$ cell lines and primary $\mathrm{PCa}$ tissues. Knockdown of KIF14 in DU145 and PC3 prostate cancer cells reduced cell proliferation, induced cell cycle arrest and apoptosis. Transcriptome analysis by RNAsequencing demonstrated that KIF4 suppression led to transcriptional changes of genes involved in p53 and TGF-beta signaling pathway. In addition, gene expression of a few of cell cycle regulated proteins (GADD45A, GADD45B, p21 and CCNB1) and apoptotic proteins (PIDD and SHISA5) were confirmed by quantitative real-time PCR. We further found that KIF14 expression was significantly upregulated in primary human PCa specimens. KIF14 protein level was positively correlated with clinical stage and Gleason Score. Patients with higher KIF14 expression had shorter 5-year overall survival time than those with lower KIF14 expression. Thus, our data indicate that KIF14 overexpression contributes to prostate tumor progression and is associated with poor prognosis for PCa patients, which could be served as a novel and useful prognostic indicator for $\mathrm{PCa}$.

\section{RESULTS}

\section{KIF14 was overexpressed in PCa}

Previous studies suggested that KIF14 was increased in multiple cancer including hepatocellular carcinoma (HCC), lung cancer, breast cancer, glioma, laryngeal carcinoma and ovarian cancer [6-12]. However, the role of KIF14 in prostate cancer remains unknown yet. We first checked the gene expression of KIF14 in a serial $\mathrm{PCa}$ cell lines and normal prostate tissue and found that KIF14 was significantly upregulated in PCa cells as compared to that in normal prostate tissue (Figure 1A). We further performed a summary for KIF 14 gene expression extracted from to the Oncomine database, a cancer microarray database and Integrated Data-Mining Platform [14]. A fold-change of 2 for KIF 14 gene expression compared to control was required for criterion. Based on analysis on two datasets derived from samples of patients with PCa $[15,16]$, we determined that KIF 14 is highly overexpressed in prostate carcinoma as compared with normal prostate tissues (Figure 1B). Thus, the overexpression of KIF14 in multiple PCa cell lines and primary PCa suggested that KIF14 might be a potential oncogene in PCa.

\section{Inhibition of KIF14 leads to G2 arrest and reduced proliferation in PCa cells}

To explore the possible mechanism of KIF14 as an oncogene, we adopted a small interfering RNA (siRNA) approach to knockdown KIF14 and examined the effect of KIF14 on cell cycle distribution and proliferation. In the experimental set-up, cell lines of DU145 and PC3, where KIF14 was markedly upregulated as compared to normal control, were chosen for the functional study. The knockdown efficiency was first validated after 48 $\mathrm{h}$ transfection with siRNA. Quantitative real-time PCR analysis showed that the mRNA levels of KIF14 were reduced by $89 \pm 1 \%(P<0.01)$ and $91 \pm 5 \%(P<0.01)$ in DU145 and PC3, respectively (Figure 2A). We examined whether reduced KIF14 expression would affect prostate cancer cell growth by counting the cell numbers for three constitutive days after siRNA transfection. DU145 and PC3 cells exhibited reduced proliferation after KIF14 expression was knocked down (Figure 2B and Figure 2C). Flow cytometric analysis of propidium iodide (PI)-stained cells revealed that cells accumulated and arrested in G2 as evidenced by a significant increase in the fraction of cells in G2 in siKIF14-trasnfected DU145 and PC3 cells as compared to that in scramble control transfected cells (Figure 2D and Figure 2E), indicating that loss of KIF 14 induces $\mathrm{G} 2$ arrest in $\mathrm{PCa}$.

\section{Knockdown of KIF14 leads to apoptosis of PCa cells}

We further examined the effect of KIF14 on apoptosis. Flow cytometric analysis of Annexin V-PE/7AAD-stained cells after 48 hours after siRNA transfection discovered that loss of KIF 14 produced more apoptotic cells of DU145 and PC3 (Figure 3A-3C). Taken together, these data indicate that KIF 14 overexpression 

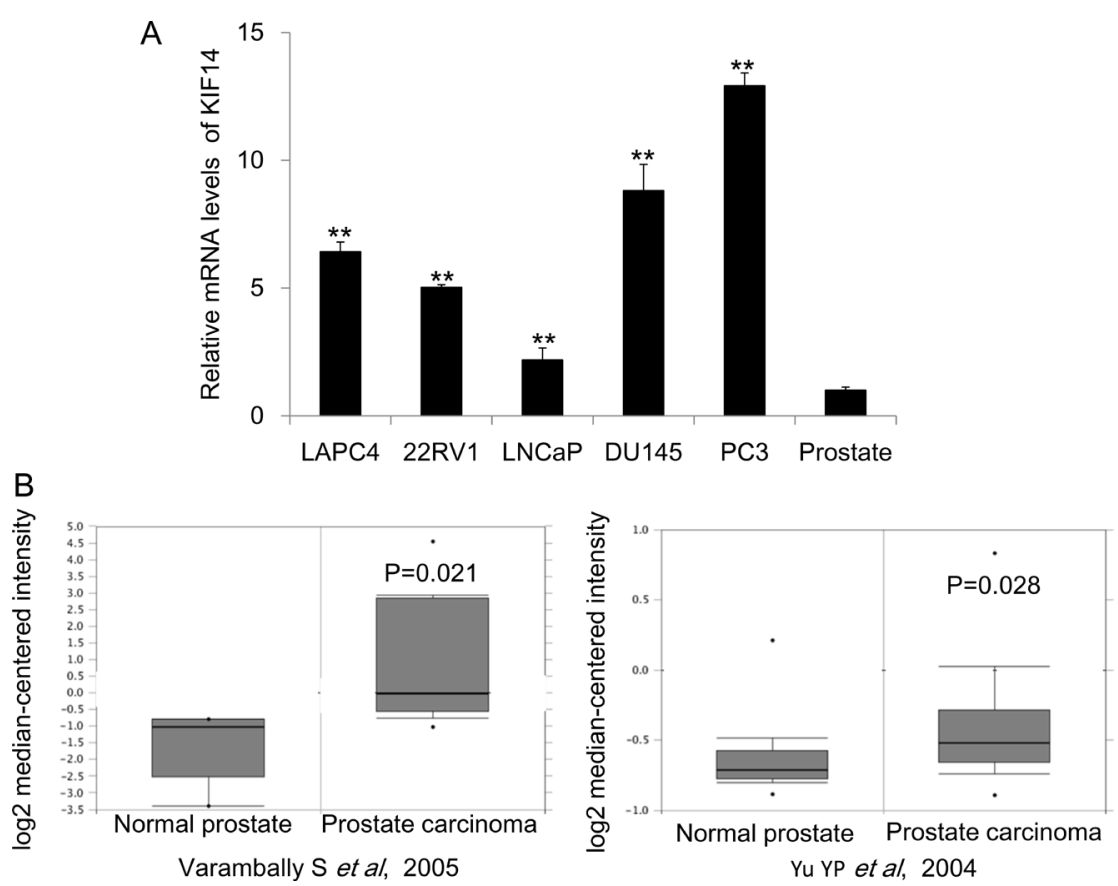

Figure 1: Expression levels of KIF14 in prostate cancer. (A) Expression levels of KIF14 in a serial of prostate cancer cell lines and normal prostate tissue. ${ }^{*} P<0.05,{ }^{* *} P<0.01$. (B) Increased expression of KIF14 in studies by Varambally et al. (left) and Yu et al. (right) as obtained from the Oncomine cancer database.
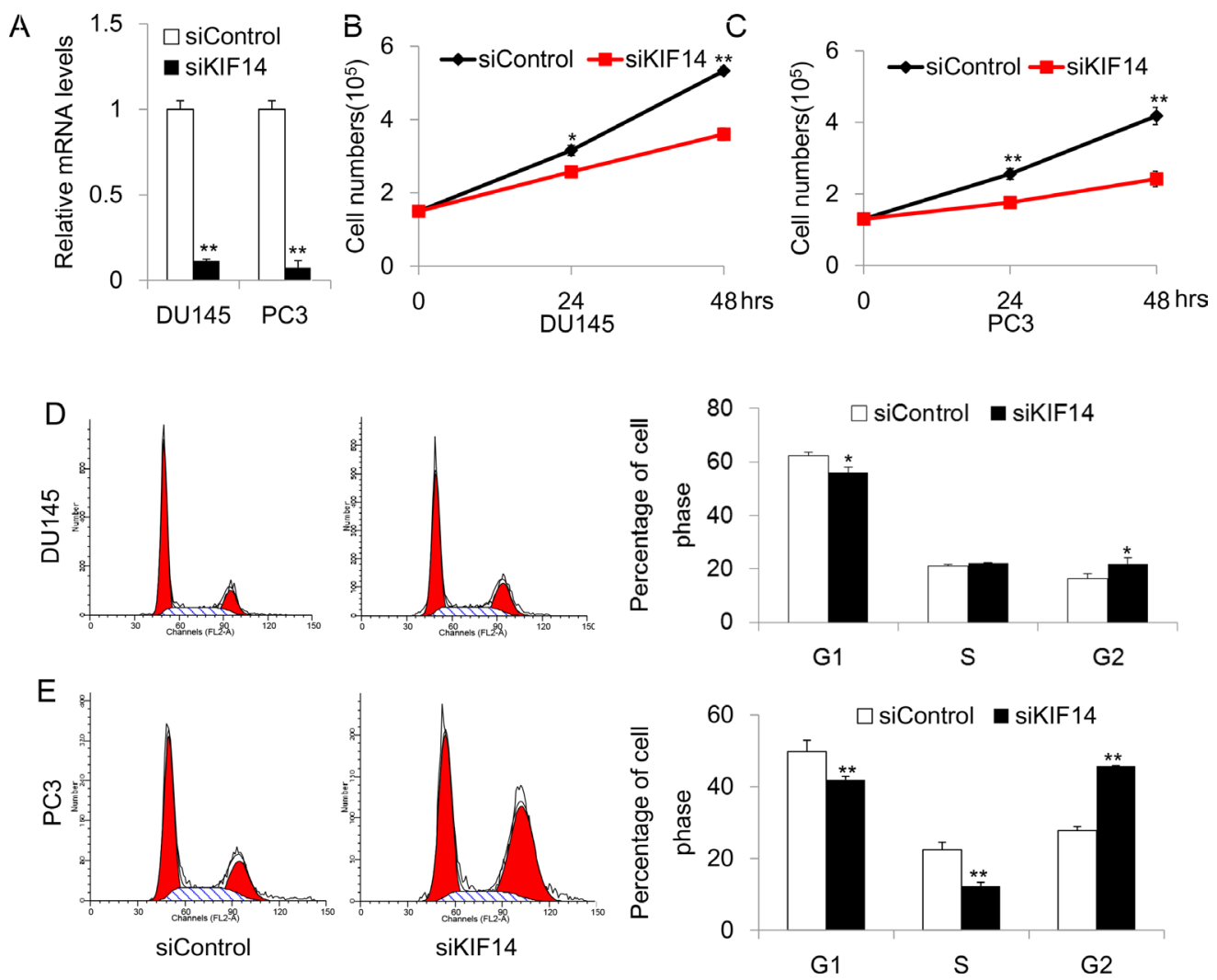

Figure 2: Knockdown of KIF14 inhibits cell proliferation and induce G2 arrest in DU145 and PC3 cell lines. (A) Quantitative real-time PCR analysis of knockdown efficacy of KIF14 in DU145 and PC3. ${ }^{*} P<0.05,{ }^{* *} P<0.01$.(B-C) Knockdown of KIF14 resulted in G2 arrest both in DU145 and PC3 cell lines. ${ }^{*} P<0.05,{ }^{* *} P<0.01$. (D-E) Loss of KIF14 inhibits cell growth in DU145 and PC 3 cells. ${ }^{*} P<0.05,{ }^{* *} P<0.01$. 
is necessary for $\mathrm{G} 2 / \mathrm{M}$ cell cycle progression, promotion of cell proliferation, and prevention of cell apoptosis, which ultimately leads to indefinite growth of prostate cancer cells.

\section{KIF14 regulated genes involved in cell cycle and apoptosis}

Our functional study suggested that KIF14 is a potential oncogene in PCa. To explore the underlying mechanism by which KIF14 exerts oncogenic function in $\mathrm{PCa}$, we performed RNA-Sequencing analysis to identify genes that were differentially expressed in KIF14 knockdown DU145 cells and control DU145 cells. Genes with 2 fold changes were considered as significant (Figure 4A). Firstly, we analyzed the candidate genes by KEGG (Kyoto Encyclopedia of Genes and Genomes) Pathway enrichment analysis. We found that knockdown of KIF14 led to transcriptional changes of genes involved in $\mathrm{p} 53$, TGF-beta, Hippo and PI3K-AKT signaling pathways (Figure 4B). The involvement of these cancer related pathway indicated that KIF14 has functional role in carcinogenesis of $\mathrm{PCa}$. We further confirmed the expression of potential KIF14 target gene by quantitative real-time PCR. In both DU145 and PC3 cells, knockdown of KIF14 upregulated the gene expression of GADD45A, GADD45B, p21, PIDD and Shisa5, which contribute to growth arrest and apoptosis induction, while downregulated $C C N B 1$ that promotes cell cycle progression (Figure 4C and Figure 4D). Our data suggested that KIF14 may function as a candidate oncogene through regulating genes involved in cell cycle modulation and apoptosis, thus leading to tumor progression in $\mathrm{PCa}$.

\section{KIF14 was overexpressed in primary $\mathrm{PCa}$ and positively correlated with clinical stage in patients with PCa}

Our study suggested that KIF14 was upregulated in PCa cell lines. We further investigated whether KIF14 was overexpressed in primary human $\mathrm{PCa}$ specimens by immunohistochemical staining. KIF14 was highly expressed in 57\% (37/65) primary PCa tissues, while it was either absent or weakly expressed in another $20 \mathrm{BPH}$ patients (20/25) (Table 1). Thus, KIF14 was significantly overexpressed in primary human $\mathrm{PCa}(P=0.0017$, Table 1). To further clarify the relationship between KIF14 expression and PCa progression, we investigated its expression and clinicopathological variables in 65 patients (Table 2). KIF14 expression was positively correlated with histological grade (Figure 5A). Moreover, KIF14 expression was significantly correlated with clinical stage $(P=0.0002)$ and Gleason score $(P=0.0041)$. However, no correlation was found between KIF14 expression and age and metastasis.

\section{Prognostic significance of KIF4 expression}

Clinical Gleason Score 8-10 is a negative independent prognostic factor on the progression free survival. In the present study, whereas 36\% (5/14) of patients with Gleason Score $\leq 6$ showed low KIF14 expression, 75\% (21/28) of
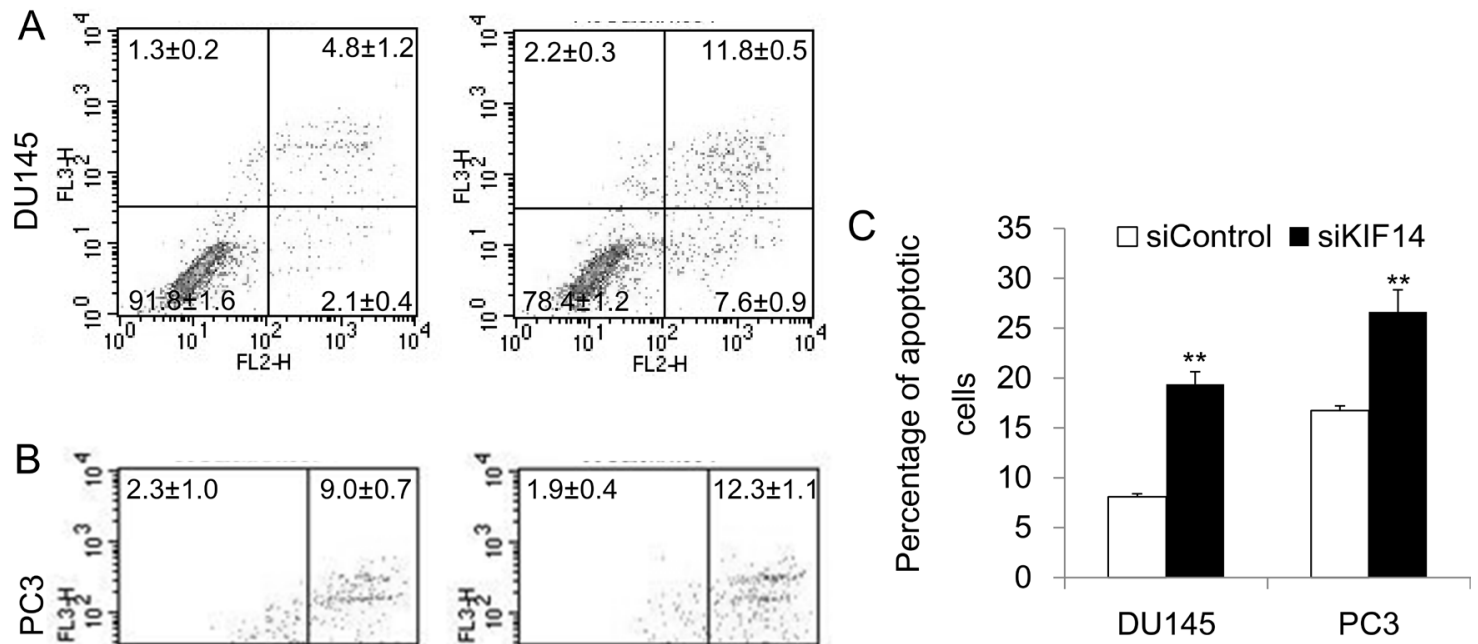

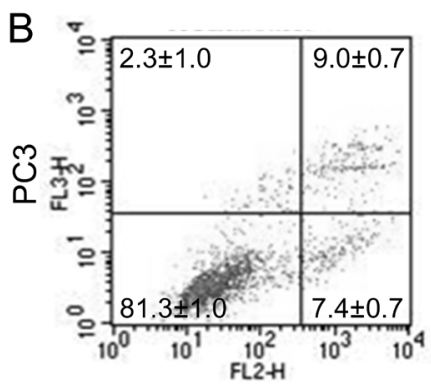

siControl

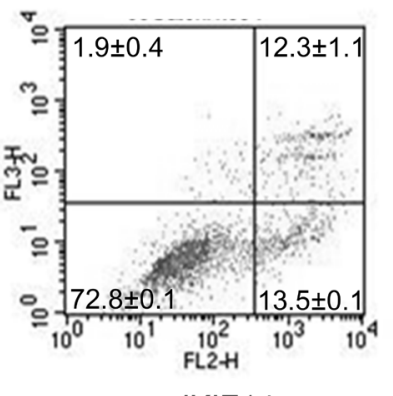

siKIF14

Figure 3: Knockdown of KIF14 induces apoptosis. (A-B) Knockdown of KIF14 resulted in apoptosis both in DU145 and PC3 cell lines as determined by flow cytometric analysis of Annexin V-PE/7-AAD-stained cells. (C) Statistical results of percentage of apoptotic cells in DU145 and PC3 cell lines. ${ }^{*} P<0.05,{ }^{* *} P<0.01$. 
Table 1: Expression of KIF14 in BPH and PCa tissues

\begin{tabular}{lcccc}
\hline Type & Case Number & Low Expression & High Expression & $P$ \\
\hline $\mathrm{BPH}$ & 25 & 20 & 5 & 0.0017 \\
$\mathrm{PCa}$ & 65 & 28 & 37 & \\
\hline
\end{tabular}

In 20/25 BPH specimens, KIF14 was either absent or weakly expressed. However, the PCa tissue samples (37/65) were scored strong positive or moderate positive expression. The difference was significant. $(P<0.05)$.
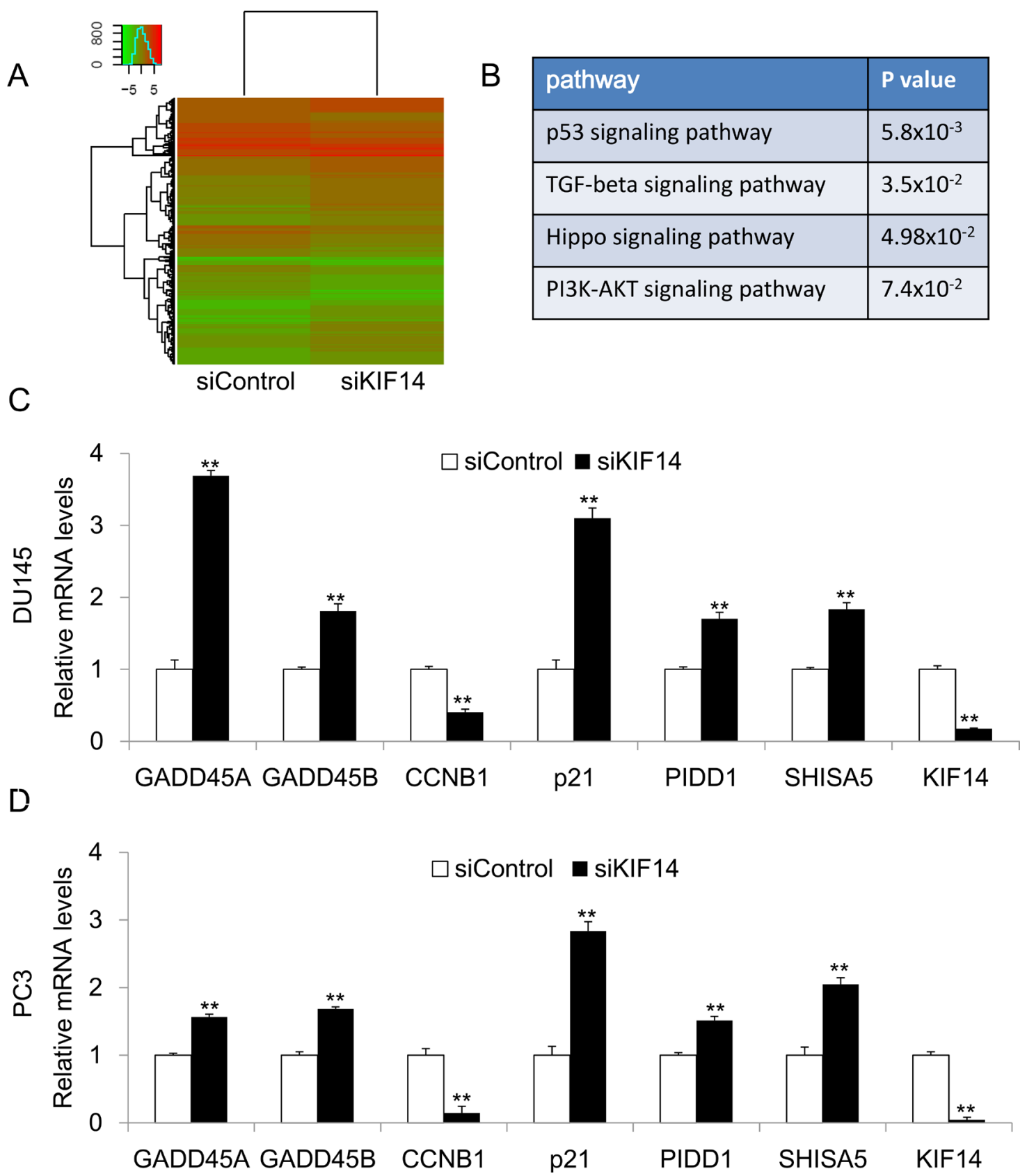

Figure 4: KIF14 downstream gene analysis. (A) Heatmap of RNA-sequencing results from KIF14 knockdown DU145 cells and control DU145 cells. (B) KEGG Pathway enrichment analysis for RNA sequencing analysis. (C-D) Confirmation of differentially expressed target genes in DU145 and PC3 cells by quantitative real-time PCR. ${ }^{*} P<0.05,{ }^{* *} P<0.01$. 
men with Gleason Score of 8-10 displayed high KIF14 expression ( $P=0.0041$, Table 2$)$, indicating a positive correlation of KIF14 overexpression with poor prognosis. Moreover, Kaplan-Meier analysis demonstrated that patients with higher KIF14 expression had a significantly shorter 5-year overall survival time (Figure 5B). Thus, these data emphasize the close association between KIF14 expression and prognosis in patients with PCa.

\section{DISCUSSION}

Tumorigenesis is a complex process which contains lots of abnormal disruptions of tumor suppressor genes, oncogenes and their related signaling pathways [17-26]. $\mathrm{PCa}$ is one of the most common non-cutaneous malignancies among men worldwide with a peak incidence in men of $\sim 70$ years [27]. Though some of the prostate cancer patients are no need to exposure to treatment, about one-third of patients with organ-confined PCa fail surgical treatment and progress to aggressive or metastatic lethal disease in 10 years [2]. Therefore, understanding mechanisms underlying the tumorigenesis and development of $\mathrm{PCa}$ is needed for early diagnosis, risk assessment and selection of therapeutic strategies against PCa. In this study, we identified KIF14 as a potential candidate oncogene involved in tumor progression and poor prognosis in prostate cancer.
KIF14 is a member of kinesin family proteins involved in cell division, microtubule polymer dynamics, intracellular transportation and signal transduction [3]. There is increasing evidence that KIF14 is overexpressed in multiple types of tumors, including hepatocellular carcinoma, lung cancer, breast cancer, etc [6-12], implicating its role as an potential oncogene. The upregulation of KIF14 might be caused by genomic amplification $[6,9,12]$. It was reported that in hepatocellular carcinoma, loss of KIF14 downregulates the expression of Skp2 and Cks1, leading to accumulation of p27Kip1. Downregulation of Skp2 and Cks1 also resulted in cytokinesis failure, which may inhibit tumor growth [6]. Inhibition of KIF14 inhibited the migration and induces apoptosis via inactivation of Akt kinase in HCC cells [7]. The effect of KIF14 on AKT phosphorylation was also reported in triple-negative breast cancer [9]. However, the expression of KIF14 and its role in prostate carcinoma remain unclear thus far. The present study demonstrated for the first time, to our knowledge that KIF14 is a candidate oncogene in $\mathrm{PCa}$, which is upregulated in multiple cell lines of PCa and primary human PCa tissues. Remarkly, KIF14 depletion induced cell cycle arrest, reduced proliferation and apoptosis in $\mathrm{PCa}$ cells at least partially through upregulating $G A D D 45 A, G A D D 45 B$, p21, PIDD and Shisa5, which contribute to growth arrest
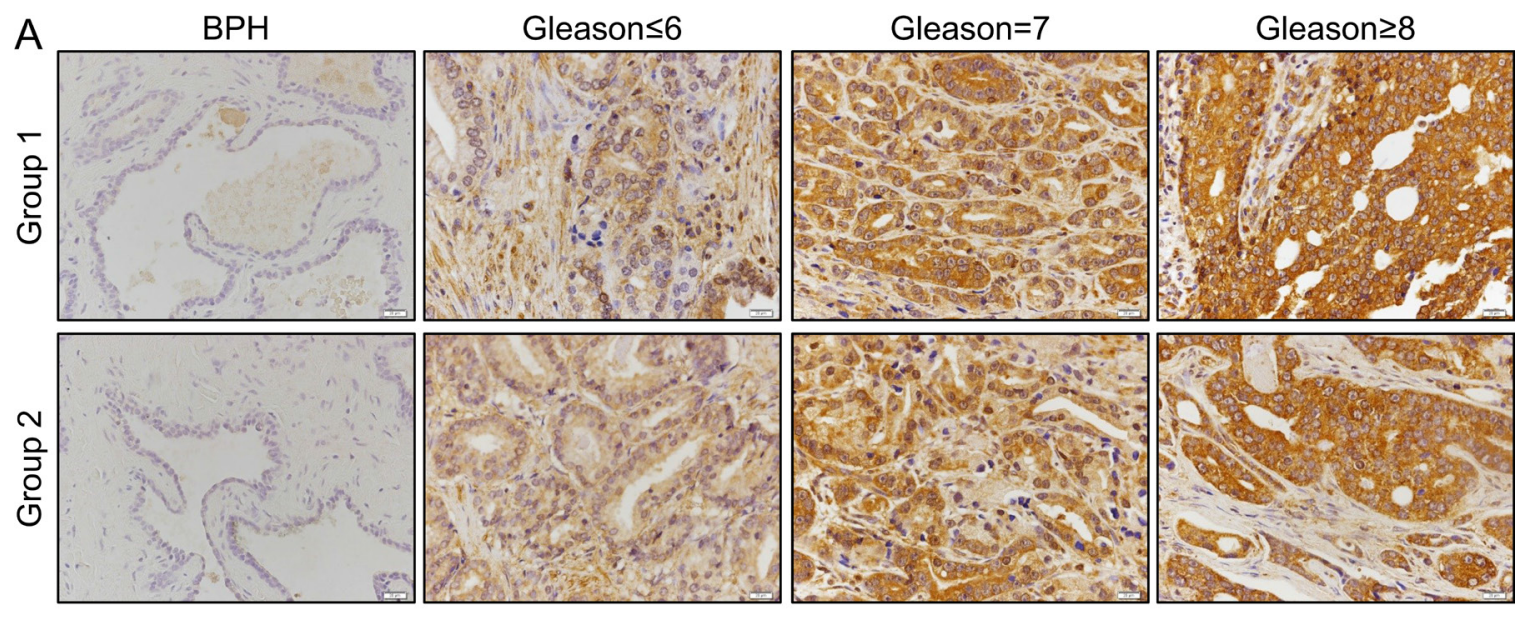

B

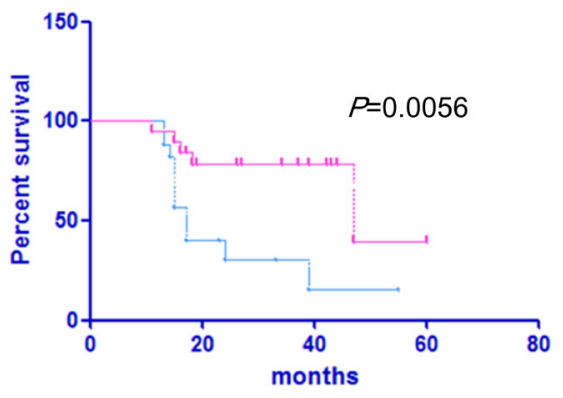

+ KIF14 low expression $n=19$

$\rightarrow \mathrm{KIF} 14$ high expression $\mathrm{n}=17$

Figure 5: IHC analysis of KIF14 in prostate cancer patient samples. (A) Representative IHC image of KIF14 in primary PCa samples. KIF14 was upregulated in patient samples and positively correlated with histological grade of primary PCa. (B) Kaplan-Meier survival curve for the correlation of KIF14 expression and 5-year overall survival of patients with PCa. Survival analysis was performed for KIF14 in a cohort of 19 low expression patients and 17 high expression patients, using a log-rank test $P=0.0056$. 
Table 2: Relationship between clinicopathological variables and KIF14 expression level in PCa patients

\begin{tabular}{|c|c|c|c|c|}
\hline Classification & Number & Low expression, $n$ & High expression, $n$ & $\boldsymbol{P}$ \\
\hline Age (year) & & & & 0.7171 \\
\hline$<60$ & 8 & 4 & 4 & \\
\hline$\geq 60$ & 57 & 24 & 33 & \\
\hline Primary tumor & & & & 0.0002 \\
\hline $\mathrm{T} 1 \sim \mathrm{T} 2$ & 31 & 22 & 12 & \\
\hline $\mathrm{T} 3 \sim \mathrm{T} 4$ & 34 & 6 & 25 & \\
\hline Metastasis & & & & 0.1232 \\
\hline- & 47 & 23 & 24 & \\
\hline+ & 18 & 5 & 13 & \\
\hline Gleason Score & & & & 0.0041 \\
\hline$\leq 6$ & 14 & 9 & 5 & \\
\hline 7 & 23 & 12 & 11 & \\
\hline$\geq 8$ & 28 & 7 & 21 & \\
\hline
\end{tabular}

low expression including no(-) and weak $(+)$ staining, high expression including moderate $(++)$ and strong $(+++)$ staining.

Metastasis including lymph node and Distant metastasis.

and apoptosis induction, and downregulating $C C N B 1$ that promotes cell cycle progression.

GADD45 mediated G2/M checkpoint in human and murine cells [28], this effect may be due to GADD45 ability to dissociate complexes of Cyclin B1 and Cdc2 [29]. The dephosphorylation of the CDC2/ CCNB complex mediated by Cdc 25 allows cells entry into $M$ phase $[30,31]$. The main function of $\mathrm{p} 21$ in cell cycle regulation is inhibiting the activity of cyclin $\mathrm{B} /$ $\mathrm{Cdk} 1$ complexes. Thus $\mathrm{p} 21$ is responsible for the nuclear retention of the cyclin $\mathrm{B} / \mathrm{Cdk} 1$ complex and cyclin $\mathrm{B}$ degradation in response to DNA damage [32]. In our study, we found CCNB was repressed, while GADD45A, GADD45B and p21 were upregulated after KIF14 inhibition. The changed expression profile of these genes could explain G2 arrest in KIF14 knockdown cells. G2 arrest will in turn lead to lower proliferation rate in cells and it could explain the inhibition of proliferation in KIF14 knockdown cells. We also found that SKP2 was downregulated in our microarray study (data not shown), which is consistent with other group's study [6]. We also found that PIDD, which interact with other death domain proteins and function as an adaptor protein in cell death-related signaling processes [33], was upregulated in KIF14 downregulated cells. PIDD is an effector of p53-induced apoptosis and it could act as switch between cell survival and cell death in response to DNA damage [33]. Shisa5 is another apoptotic protein upregulated in KIF14 knockdown cells. Shisa5 is localized to the endoplasmic reticulum and together with p53 induces apoptosis in a caspase-dependent manner $[34,35]$. The upregulation of these two proteins will induce apoptosis in KIF14 knockdown cells. Our future study will be focused on how KIF14 regulates those genes' expression.

Except for KIF14, many other kinesin proteins are reported as oncoproteins [36-43]. For example, KIFC1 induces resistance to docetaxel and is associated with survival of patients with prostate cancer [36]; KIF11 is a driver of invasion, proliferation, and self-renewal in glioblastoma [37]; KIF3C promotes tumor growth and metastasis in breast cancer by regulating TGF- $\beta$ signaling [38]; It is also reported that inhibition of KIF22 suppresses cancer cell proliferation by delaying mitotic exit through upregulating CDC25C expression [39]; KIF3a can promote proliferation and invasion via Wnt signaling in advanced prostate cancer [40]. All these further support our finding that KIF14 is a functional oncoprotein in prostate cancer.

It has been reported that KIF14 is a marker of poor prognosis in patients with hepatocellular carcinoma and ovarian cancer, where KIF14 overexpression enhances tumor growth, while its knockdown decreases tumorigenicity in vitro and in xenografts [13]. However, the role of KIF14 as an indicator in PCa is largely unknown. Here, we found that KIF14 was upregulated in most clinical PCa tissues and cell lines. Subsequently, by immunohistochemical methods, we observed a positive 
correlation between KIF4 expression and histological grade, clinical stage and Gleason Score. Kaplan-Meier analysis demonstrated that KIF14 was a prognostic factor for patient survival. Our results thus indicate that KIF14 could be served as a novel and useful prognostic biomarker for PCa.

In summary, our study found that KIF14 is upregulated in PCa cell lines and clinical PCa tissues. Inhibition of KIF14 suppresses cell proliferation, induces G2 arrest and apoptosis. This effect was mainly caused by regulating GADD45A, GADD45B, CCNB, p21, PIDD and SHISA5 expression. Moreover, the protein level of KIF14 is positively correlated with histological grade, clinical tumor stage and poor prognosis in patients with PCa. Thus, our results suggest that KIF14 could act as a candidate oncogene that contributes to tumor progression and poor prognosis in PCa. KIF14 may represent a novel molecular target for the treatment of PCa.

\section{MATERIALS AND METHODS}

\section{Cell culture and transfection}

, DU145 and PC3) were used for this study. All cells were purchased from ATCC. Cells were maintained in RPMI 1640 medium (Invitrogen, Carlsbad, CA) containing $10 \%$ fetal bovine serum (FBS), $1 \%$ penicillin-streptomycin (10 ng/ml penicillin and $10 \mathrm{U} / \mathrm{ml}$ streptomycin), and 2.5 $\mathrm{mM}$ glutamine at $37^{\circ} \mathrm{C}$ in a humidified $5 \% \mathrm{CO} 2$ incubator [44]. KIF14-short interfering RNA (siKIF14, Santa Cruz biotechnology, catalog no: sc-60882) and control siRNA (siControl, Santa Cruz biotechnology, catalog no: sc37007) were purchased from Santa Cruz Biotechnology (Dallas, TX, USA). Transfection was carried out according to the manufacturer's instruction using RNAiMAX transfection reagent (Invitrogen, Eugene, OR, USA, catalog no: 13778).

\section{RNA extraction, reverse transcription and quantitative realtime PCR}

RNA was extracted with Trizol reagent according to manufacturer's protocol (Invitrogen, Eugene, OR, USA). One normal prostate RNA was purchased from Clontech (Clontech, Palo Alto, CA). Reverse transcription was performed with Random hexamers and SuperScriptIII (Invitrogen, Eugene, OR, USA). Quantitative realtime PCR was carried out with the Applied Biosystems 7300 real-time systems using real-time PCR Master Mix (SYBR Green). The standard curve was used to establish amplification efficiency [45]. All primers used are listed in Table 3. Each experiment was performed in triplicate in three independent experiments.

\section{Growth curve}

DU145 or PC3 cells were seeded in six-well plates at an appropriate density with penicillin/streptomycin free medium. Cells were transfected with control siRNA or siKIF14, respectively. After transfection, cell numbers were counted every 24 hours including the start point. Each experiment was conducted in triplicate in three independent experiments.

\section{Flow cytometry analysis of cell cycle}

About 48 hours after transfection, cells were harvested and fixed with ice-cold $70 \%$ ethanol for 24 hours. After that, cells were washed with PBS once and stained with $100 \mu \mathrm{l}$ of $50 \mathrm{mg} / \mathrm{L}$ propidium iodide (PI) for $15 \mathrm{~min}$ at $4^{\circ} \mathrm{C}$ in dark. The cell cycle profiles were assayed by flow cytometry (excitation at $536 \mathrm{~nm}$ and emission at $617 \mathrm{~nm}$ ) with a flow cytometer (FACS Calibur ${ }^{\mathrm{TM}}$, BD Biosciences, CA, USA) and data were analyzed with the ModFit LT software. Each experiment was conducted in triplicate in three independent experiments.

\section{Apoptosis assay}

Apoptosis was assessed by flow cytometry analysis. Apoptotic cells were detected with PE Annexin V Apoptosis Detection Kit I (BD Bioscience). About 48 hours after transfection, cells were harvested and centrifuged at $1000 \mathrm{rpm}$ for $10 \mathrm{~min}$, rinsed with cold PBS once. About $1 \times 10^{5}$ cells were stained with $5 \mu \mathrm{PE}$ Annexin V and $5 \mu 17$-AAD working solution for $15 \mathrm{~min}$ at room temperature in dark. The ratio of apoptotic cells were analyzed by flow cytometry. The total number of early apoptotic cells and late apoptotic cells were considered as total apoptotic cells. Each experiment was conducted in triplicate in three independent experiments.

\section{RNA sequencing}

RNA was extracted with an RNeasy Mini kit (QIAGEN). Firstly, mRNA was purified and RNASequencing library preparation was performed according to the manual of manufacturers (KAPA biosystems). Sequencing reactions were performed with the Illumina HiSeq platform. RNA-seq reads were mapped to the human genome (hg19) using Burrows-Wheeler Aligner (bwa). The duplicate reads were then marked by picard. The HTseq tool was used to calculate the reads count for each gene. Finally, we used the Reads Per Kilobase per Million mapped reads (rpkm) command in edgeR package to calculate the rpkm of each gene. Specifically, we added one to the reads count for each gene and take log values. These log values were inputted into the heatmap. 2 in R package gplots to generate the heatmap figure [46]. The genes with 2 fold changes were loaded to David bioinformatics database (https:// david.ncifcrf.gov/) for pathway analysis.

\section{Oncomine analysis}

The Oncomine cancer microarray database (http:// www.oncomine.com) [14] was used to analyze expression 
Table 3: Primer list used in this study

\begin{tabular}{|c|c|c|c|}
\hline Application & Primer name & Sequence $\left(5^{\prime} \rightarrow 3^{\prime}\right)$ & Size (bp) \\
\hline \multirow{16}{*}{ Realtime PCR } & KIF14F & TTCAGAACACCTCTGCAGGA & \multirow[t]{2}{*}{$128 \mathrm{bp}$} \\
\hline & KIF14R & ACTCATGAAGACTACCTGGG & \\
\hline & GAPDHF & TCATTGACCTCAACTACATG & \multirow[t]{2}{*}{$131 \mathrm{bp}$} \\
\hline & GAPDHR & TCGCTCCTGGAAGATGGTGAT & \\
\hline & $\mathrm{P} 21 \mathrm{~F}$ & AGACCAGCATGACAGATTTC & \multirow[t]{2}{*}{$140 \mathrm{bp}$} \\
\hline & $\mathrm{P} 21 \mathrm{R}$ & ACTGAGACTAAGGCAGAAGA & \\
\hline & $\mathrm{CCNBF}$ & CCTCCGGTGTTCTGCTTCTC & \multirow[t]{2}{*}{$122 \mathrm{bp}$} \\
\hline & CCNBR & GCCTGCCATGTTGATCTTCG & \\
\hline & GADD45AF & CTTGGAGACCGACGCTGG & \multirow[t]{2}{*}{$149 \mathrm{bp}$} \\
\hline & GADD45AR & TGTAGCGACTTTCCCGGC & \\
\hline & GADD45BF & ATCAACATCGTGCGGGTGT & \multirow[t]{2}{*}{$122 \mathrm{bp}$} \\
\hline & GADD45BR & GTGTGAGGGTTCGTGACCAG & \\
\hline & PIDD1F & TGTTCGAGGGCGAAGAGTTC & \multirow[t]{2}{*}{$148 \mathrm{bp}$} \\
\hline & PIDD1R & TCCAGAGTGGTGGTCACGTA & \\
\hline & SHISAF & GAAAGGTGTGCTGTGCCTGA & \multirow[t]{2}{*}{$105 \mathrm{bp}$} \\
\hline & SHISAR & TGACATGGGGTCGTTGTAGC & \\
\hline
\end{tabular}

profiles of KIF14 in a variety of human prostate cancer and normal tissues. Two representative prostate cancer datasets $[15,16]$ were presented in this study. A foldchange of 2 for KIF14 gene expression compared to control was required for criterion.

\section{Patient samples and IHC}

All patient samples were collected in Shenzhen People's Hospital with the permission of patients. All $\mathrm{PCa}$ and benign prostatic hyperplasia (BPH) specimens were obtained immediately after operation (radical prostatectomy, transrectal biopsy or transurethral resection of the prostate). Tissues were then fixed in $10 \%$ buffered formalin and embedded in paraffin. Tissue sections were stained with KLF14 antibody purchased from abcam. The protocol of IHC staining was described before [47]. The clinical characteristics of the PCa cohort are listed in Table 2. The tumor staging and differentiation of PCa was defined according to the 2010 AJCC Cancer Staging Manual seventh edition. (Well differentiation: Gleason Score $\leq 6$ ) Moderate differentiation: Gleason Score $=7$; Poor differentiation: Gleason Score $\geq 8$ )

\section{Follow-up}

All patients with $\mathrm{PCa}$ received check-ups every 2-3 months during the first 2 years and every 3-6 months there after until the follow-up period ended in January 2017. Surgeons who were blinded to the study performed the follow-up study. The overall survival was defined as the length of time between surgery and either the death of the patient or the last follow-up visit.

\section{Statistical analysis}

Data are presented as mean \pm standard deviation. Statistical assessments were carried out using Student's $t$ test. $P<0.05$ was considered statistically significant.

\section{ACKNOWLEDGMENTS AND FUNDING}

This work was supported by the Shenzhen Commission of Science and Innovation program (JCYJ20150403101028172; JCYJ20160422091523612; GCZX2015043016165448), National Natural Science Foundation of China (81301783; 81670760; 31500940), Shenzhen Dedicated Funding for Strategic Development of Emerging Industry (JCYJ20140418091413584) and funding from Guangdong Provincial Department of Science and Technology (2014A020212370)

\section{CONFLICTS OF INTEREST}

No potential conflicts of interest were disclosed.

\section{REFERENCES}

1. Siegel RL, Miller KD, Jemal A. Cancer statistics, 2016. CA Cancer J Clin. 2016; 66:7-30.

2. Westdorp H, Skold AE, Snijer BA, Franik S, Mulder SF, Major PP, Foley R, Gerritsen WR, de Vries IJ. 
Immunotherapy for prostate cancer: lessons from responses to tumor-associated antigens. Front Immunol. 2014; 5:191.

3. Miki H, Okada Y, Hirokawa N. Analysis of the kinesin superfamily: insights into structure and function. Trends Cell Biol. 2005; 15:467-476.

4. Carleton M, Mao M, Biery M, Warrener P, Kim S, Buser C, Marshall CG, Fernandes C, Annis J, Linsley PS. RNA interference-mediated silencing of mitotic kinesin KIF14 disrupts cell cycle progression and induces cytokinesis failure. Mol Cell Biol. 2006; 26:3853-3863.

5. Gruneberg U, Neef R, Li X, Chan EH, Chalamalasetty RB, Nigg EA, Barr FA. KIF14 and citron kinase act together to promote efficient cytokinesis. J Cell Biol. 2006; 172:363-372.

6. Xu H, Choe C, Shin SH, Park SW, Kim HS, Jung SH, Yim SH, Kim TM, Chung YJ. Silencing of KIF14 interferes with cell cycle progression and cytokinesis by blocking the p27(Kip1) ubiquitination pathway in hepatocellular carcinoma. Exp Mol Med. 2014; 46:e97.

7. Yang T, Zhang XB, Zheng ZM. Suppression of KIF14 expression inhibits hepatocellular carcinoma progression and predicts favorable outcome. Cancer Sci. 2013; 104:552-557.

8. Corson TW, Zhu CQ, Lau SK, Shepherd FA, Tsao MS, Gallie BL. KIF14 messenger RNA expression is independently prognostic for outcome in lung cancer. Clin Cancer Res. 2007; 13:3229-3234.

9. Singel SM, Cornelius C, Zaganjor E, Batten K, Sarode VR, Buckley DL, Peng Y, John GB, Li HC, Sadeghi N, Wright WE, Lum L, Corson TW, et al. KIF14 promotes AKT phosphorylation and contributes to chemoresistance in triplenegative breast cancer. Neoplasia. 2014; 16:247-256.e2.

10. Wang Q, Wang L, Li D, Deng J, Zhao Z, He S, Zhang Y, $\mathrm{Tu}$ Y. Kinesin family member 14 is a candidate prognostic marker for outcome of glioma patients. Cancer Epidemiol. 2013; 37:79-84.

11. Markowski J, Tyszkiewicz T, Jarzab M, OczkoWojciechowska M, Gierek T, Witkowska M, Paluch J, Kowalska M, Wygoda Z, Lange D, Jarzab B. Metalproteinase ADAM12, kinesin 14 and checkpoint suppressor 1 as new molecular markers of laryngeal carcinoma. Eur Arch Otorhinolaryngol. 2009; 266:1501-1507.

12. Theriault BL, Pajovic S, Bernardini MQ, Shaw PA, Gallie BL. Kinesin family member 14: an independent prognostic marker and potential therapeutic target for ovarian cancer. Int J Cancer. 2012; 130:1844-1854.

13. Thériault BL, Corson TW. KIF14: a clinically relevant kinesin and potential target for cancer therapy. Kinesins and Cancer: Springer. 2015; 149-70.

14. Rhodes DR, Yu J, Shanker K, Deshpande N, Varambally R, Ghosh D, Barrette T, Pandey A, Chinnaiyan AM. ONCOMINE: a cancer microarray database and integrated data-mining platform. Neoplasia. 2004; 6:1-6.

15. Varambally S, Yu J, Laxman B, Rhodes DR, Mehra R, Tomlins SA, Shah RB, Chandran U, Monzon FA,
Becich MJ, Wei JT, Pienta KJ, Ghosh D, et al. Integrative genomic and proteomic analysis of prostate cancer reveals signatures of metastatic progression. Cancer Cell. 2005; 8:393-406.

16. Yu YP, Landsittel D, Jing L, Nelson J, Ren B, Liu L, McDonald C, Thomas R, Dhir R, Finkelstein S, Michalopoulos G, Becich M, Luo JH. Gene expression alterations in prostate cancer predicting tumor aggression and preceding development of malignancy. J Clin Oncol. 2004; 22:2790-2799.

17. Cheng Y, Liang P, Geng H, Wang Z, Li L, Cheng SH, Ying J, Su X, Ng KM, Ng MH, Mok TS, Chan AT, Tao Q. A novel 19q13 nucleolar zinc finger protein suppresses tumor cell growth through inhibiting ribosome biogenesis and inducing apoptosis but is frequently silenced in multiple carcinomas. Mol Cancer Res. 2012; 10:925-936.

18. Wang S, Cheng Y, Du W, Lu L, Zhou L, Wang H, Kang W, Li X, Tao Q, Sung JJ, Yu J. Zinc-finger protein 545 is a novel tumour suppressor that acts by inhibiting ribosomal RNA transcription in gastric cancer. Gut. 2013; 62:833-841.

19. Cheng Y, Geng H, Cheng SH, Liang P, Bai Y, Li J, Srivastava G, Ng MH, Fukagawa T, Wu X, Chan AT, Tao Q. KRAB zinc finger protein ZNF382 is a proapoptotic tumor suppressor that represses multiple oncogenes and is commonly silenced in multiple carcinomas. Cancer Res. 2010; 70:6516-6526.

20. Fu L, Dong SS, Xie YW, Tai LS, Chen L, Kong KL, Man K, Xie D, Li Y, Cheng Y, Tao Q, Guan XY. Down-regulation of tyrosine aminotransferase at a frequently deleted region $16 \mathrm{q} 22$ contributes to the pathogenesis of hepatocellular carcinoma. Hepatology. 2010; 51:1624-1634.

21. Shu XS, Li L, Ji M, Cheng Y, Ying J, Fan Y, Zhong L, Liu X, Tsao SW, Chan AT, Tao Q. FEZF2, a novel 3p14 tumor suppressor gene, represses oncogene EZH2 and MDM2 expression and is frequently methylated in nasopharyngeal carcinoma. Carcinogenesis. 2013; 34:1984-1993.

22. Zhang Q, Zhang L, Li L, Wang Z, Ying J, Fan Y, Xu B, Wang L, Liu Q, Chen G, Tao Q, Jin J. Interferon regulatory factor 8 functions as a tumor suppressor in renal cell carcinoma and its promoter methylation is associated with patient poor prognosis. Cancer Lett. 2014; 354:227-234.

23. Cheng Y, Tu Y, Liang P. Promoter Methylated Tumor Suppressor Genes in Glioma. Cancer Translational Medicine. 2015; 1:123-130.

24. Sun $\mathrm{S}, \mathrm{Xu}$ A, Yang G, Cheng Y. Galanin is a novel epigenetic silenced functional tumor suppressor in renal cell carcinoma. Cancer Translational Medicine. 2015; 1:183-187.

25. Chen D, Dai C, Jiang Y. Histone H2A and H2B Deubiquitinase in Developmental Disease and Cancer. Cancer Translational Medicine. 2015; 1:170-175.

26. Zhun F, Liang Y, Chen D, Li Y. Melanoma Antigen Gene Family in the Cancer Immunotherapy. Cancer Translational Medicine. 2016; 2:85-89. 
27. Siegel RL, Miller KD, Jemal A. Cancer statistics, 2015. CA Cancer J Clin. 2015; 65:5-29.

28. Wang XW, Zhan Q, Coursen JD, Khan MA, Kontny HU, $\mathrm{Yu}$ L, Hollander MC, O'Connor PM, Fornace AJ Jr, Harris CC. GADD45 induction of a G2/M cell cycle checkpoint. Proc Natl Acad Sci USA. 1999; 96:3706-3711.

29. Taylor WR, Stark GR. Regulation of the G2/M transition by p53. Oncogene. 2001; 20:1803-1815.

30. Li M, Zhang P. The function of APC/CCdh1 in cell cycle and beyond. Cell Div. 2009; 4: 2.

31. Jang SH, Kim AR, Park NH, Park JW, Han IS. DRG2 Regulates G2/M Progression via the Cyclin B1-Cdk1 Complex. Mol Cells. 2016; 39:699-704.

32. Charrier-Savournin FB, Chateau MT, Gire V, Sedivy J, Piette J, Dulic V. p21-Mediated nuclear retention of cyclin B1-Cdk1 in response to genotoxic stress. Mol Biol Cell. 2004; 15:3965-3976.

33. Cuenin S, Tinel A, Janssens S, Tschopp J. p53-induced protein with a death domain (PIDD) isoforms differentially activate nuclear factor-kappaB and caspase- 2 in response to genotoxic stress. Oncogene. 2008; 27:387-396.

34. Bourdon JC, Renzing J, Robertson PL, Fernandes KN, Lane DP. Scotin, a novel p53-inducible proapoptotic protein located in the ER and the nuclear membrane. J Cell Biol. 2002; 158:235-246.

35. Draeby I, Woods YL, la Cour JM, Mollerup J, Bourdon JC, Berchtold MW. The calcium binding protein ALG-2 binds and stabilizes Scotin, a p53-inducible gene product localized at the endoplasmic reticulum membrane. Arch Biochem Biophys. 2007; 467:87-94.

36. Sekino Y, Oue N, Shigematsu Y, Ishikawa A, Sakamoto N, Sentani K, Teishima J, Matsubara A, Yasui W. KIFC1 induces resistance to docetaxel and is associated with survival of patients with prostate cancer. Urol Oncol. 2017; 35:31.e13-20.

37. Venere M, Horbinski C, Crish JF, Jin X, Vasanji A, Major J, Burrows AC, Chang C, Prokop J, Wu Q, Sims PA, Canoll P, Summers MK, et al. The mitotic kinesin KIF11 is a driver of invasion, proliferation, and self-renewal in glioblastoma. Sci Transl Med. 2015; 7:304ra143.

38. Wang C, Wang C, Wei Z, Li Y, Wang W, Li X, Zhao J, Zhou X, Qu X, Xiang F. Suppression of motor protein KIF3C expression inhibits tumor growth and metastasis in breast cancer by inhibiting TGF-beta signaling. Cancer Lett. 2015; 368:105-114.

39. Yu Y, Wang XY, Sun L, Wang YL, Wan YF, Li XQ, Feng YM. Inhibition of KIF22 suppresses cancer cell proliferation by delaying mitotic exit through upregulating CDC25C expression. Carcinogenesis. 2014; 35:1416-1425.

40. Liu Z, Rebowe RE, Wang Z, Li Y, Wang Z, DePaolo JS, Guo J, Qian C, Liu W. KIF3a promotes proliferation and invasion via Wnt signaling in advanced prostate cancer. Mol Cancer Res. 2014; 12:491-503.

41. Duan H, Zhang X, Wang FX, Cai MY, Ma GW, Yang $\mathrm{H}$, Fu JH, Tan ZH, Fu XY, Ma QL, Wang XY, Lin P. KIF-2C expression is correlated with poor prognosis of operable esophageal squamous cell carcinoma male patients. Oncotarget. 2016; 7:80493-80507. doi: 10.18632/ oncotarget.11492.

42. Sun L, Zhang C, Yang Z, Wu Y, Wang H, Bao Z, Jiang T. $\mathrm{KIF} 23$ is an independent prognostic biomarker in glioma, transcriptionally regulated by TCF-4. Oncotarget. 2016; 7:24646-24655. doi: 10.18632/oncotarget.8261.

43. Shi C, Huang D, Lu N, Chen D, Zhang M, Yan Y, Deng L, Lu Q, Lu H, Luo S. Aberrantly activated Gli2-KIF20A axis is crucial for growth of hepatocellular carcinoma and predicts poor prognosis. Oncotarget. 2016; 7:26206-26219. doi: 10.18632/oncotarget.8441.

44. Liang P, Henning SM, Schokrpur S, Wu L, Doan N, Said J, Grogan T, Elashoff D, Cohen P, Aronson WJ. Effect of Dietary Omega-3 Fatty Acids on Tumor-Associated Macrophages and Prostate Cancer Progression. Prostate. 2016; 76:1293-1302.

45. Svec D, Tichopad A, Novosadova V, Pfaffl MW, Kubista M. How good is a PCR efficiency estimate: Recommendations for precise and robust qPCR efficiency assessments. Biomol Detect Quantif. 2015; 3:9-16.

46. Warnes GR, Bolker B, Bonebakker L, Gentleman R, Huber W, Liaw A, Lumley T, Maechler M, Magnusson A, Moeller S. gplots: Various R programming tools for plotting data. R package version. 2009; 2:1.

47. Xiao Y, Yuan Y, Zhang Y, Li J, Liu Z, Zhang X, Sheng Z, $\mathrm{Xu}$ T, Wang X. CMTM5 is reduced in prostate cancer and inhibits cancer cell growth in vitro and in vivo. Clin Transl Oncol. 2015; 17:431-437. 